\title{
Early intervention: what is it really?
}

Peta Odgers

Comcare, Canberra ACT 2600, Australia. peta.odgers@comcare.gov.au

Background: The use of early intervention (EI) strategies following workplace injuries and illnesses have long been espoused by medical specialists, rehabilitation providers, regulators and others to be important in hastening recovery, and shortening the length of absence from work. Little is known, however, about the application, content or effectiveness of EI practices within the Comcare scheme.

Objectives: The aim of this research is to provide a strategic assessment of EI in the Comcare scheme by:

- investigating what constitutes EI from an employer perspective,

- determining which agencies/organisations use EI and why, and

- establishing the impact EI has or could have on the scheme.

Methods: Phase one will consist of in-depth interviews and/or small group discussions with representatives from both premium paying and self-insured organisations. An independent research company will conduct phase one on behalf of Comcare between February and March 2014. A targeted sampling approach has been used to ensure organisations with known greater and lesser experience in the use of EI are included. Phase two will build on phase one, using an online survey to collect information from all premium paying and self-insured organisations in the Comcare Scheme.

Findings: It is anticipated that, within the Comcare scheme, the project will identify: what constitutes EI; what role EI plays or could play; what influences EIs success; and how EI is impacting the type and complexity of claims entering the scheme.

Discussion: This paper will focus on the key findings of the research and the implications they have for policies, practices and service delivery.

Conclusion: Understanding the role of EI in organisations provided Comcare with a framework to better guide and support employers in their response to workplace injuries and illnesses, and improving the outcomes for their people. 